\title{
Endoscope Calibration and Accuracy Testing for 3D/2D Image Registration
}

\author{
Rasool Khadem ${ }^{1}$, Michael R. Bax ${ }^{2}$, Jeremy A. Johnson ${ }^{2}$, Eric P. Wilkinson ${ }^{2}$ \\ and Ramin Shahidi ${ }^{2}$ \\ ${ }^{1}$ CBYON, 2275 East Bayshore Road \#101, Palo Alto, CA 94303, USA \\ rasool@cbyon.com \\ ${ }^{2}$ Image Guidance Laboratories, 300 Pasteur Drive \#S-012, Stanford, CA 94305-5327, USA \\ http://igl. Stanford.edu
}

\begin{abstract}
New surgical navigation techniques incorporate the use of live surgical endoscope video with 3D reconstructed MRI or CT images of a patient's anatomy. This image-enhanced endoscopy requires calibration of the endoscope to accurately the register the real endoscope video to the virtual image. The calibration and accuracy testing of such a system and a simple yet effective linear method for lens-distortion compensation are described.
\end{abstract}

\section{Background and Theory}

Today lightweight endoscopes are used in small body cavities, but they display only visible surfaces and are unable to view the interior of opaque tissue. Combining endoscopic video with overlaid volumetrically-reconstructed CT or MRI patient images permits surgeons to look beyond visible surfaces and provides "on-the-fly" $2 \mathrm{D}$ and 3D information for planning and navigation [1]. Precise endoscope calibration and exhaustive accuracy testing are necessary to ensure surgical quality.

Following calibration, tracking the position and rotation of the endoscope enables the rendering of virtual images to match the endoscope video. A ray from the center of projection (the optical origin) to a point in physical space passes through the image projection plane (the $\mathrm{CCD}$ ). Errors such as residual lens distortion or tracking error cause this intersection to be some distance $e$ from the image point, as in Fig. 1(a).

Adding full non-linear radial lens-distortion compensation to the rendering engine causes a drop in performance; an alternative method, here named "constant-radius linear compensation", is to linearly scale the virtual image such that the radii of the predefined region of interest in each image are made equal.

\section{Method}

A STORZ Tricam endoscope with a 50200A telescope, Traxtal universal passive trackers, and an NDI Polaris hybrid optical tracking system were used.

Tsai's calibration algorithm [2] was used. The calibration target was a planar grid pattern of black dots 1 and $2 \mathrm{~mm}$ in diameter, with the grid rows and columns spaced W. Niessen and M. Viergever (Eds.): MICCAI 2001, LNCS 2208, pp. 1361-1362, 2001.

(c) Springer-Verlag Berlin Heidelberg 2001 
$3 \mathrm{~mm}$ apart. This was mounted in a jig with an attached tracker such that the telescope of the endoscope in calibration is positioned $15 \mathrm{~mm}$ away from the center of the target and angled $30^{\circ}$ from the normal of the target plane.

The physical target for accuracy testing was a version of the calibration target large enough to fill the field of view (FOV) of the endoscope at a distance of $65 \mathrm{~mm}$, and mounted on a tracked moveable plate. The grid position was localized relative to the tracker. The grid was stepped through a set of distances from 5 to $65 \mathrm{~mm}$ from the tip of the endoscope's telescope. At each position the image error $e$ was found for each dot in the endoscope video image. This was repeated for 20 separate calibrations.

\section{Results}

Fig. 1(b) shows the mean error between the rendered location of the dots in the virtual image and the corresponding dots in the endoscope video image, normalized to the diameter of the FOV, as a function of distance from the center of the image. Fig. 1(c) shows the normalized error as a function of distance of the target plane from the endoscope tip. It can be seen that constant-radius linear compensation is an effective scheme for overcoming lens distortion without a rendering performance loss.

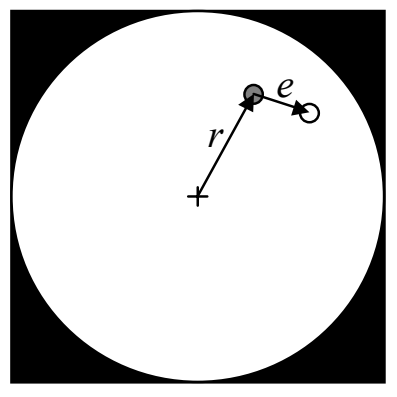

(a)

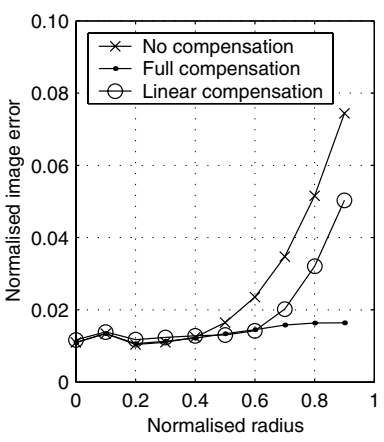

(b)

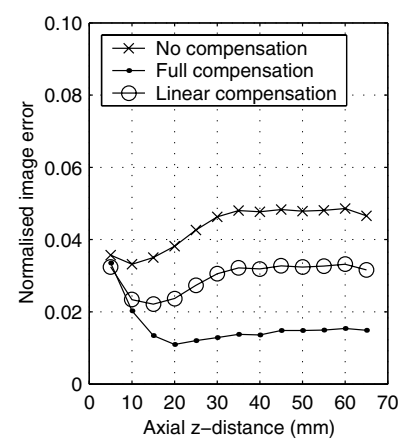

(c)

Fig. 1. (a) Image space error: a point in the video image (gray dot) and the projection ray intersection with the image plane (white dot). (b) Mean image error versus image radius and (c) mean image error versus target plane distance, normalized to the FOV diameter.

\section{References}

1. D.J. Vining, Virtual Endoscopy: Is It Reality? Radiology 200 (1996) 30-31

2. Tsai, R.Y.: An Efficient and Accurate Camera Calibration Technique for 3D Machine Vision. Proc. of the IEEE Conference on Computer Vision and Pattern Recognition (1986) 364-374

3. Khadem, R., Yeh, C., Sadeghi-Tehrani, M., Bax, M.R., Johnson, J.A., Welch, J.N., Wilkinson, E.P., Shahidi, R.: Comparative Tracking Error Analysis of Five Different Optical Tracking Systems. Computer Aided Surgery 5 (2000) 98-107 